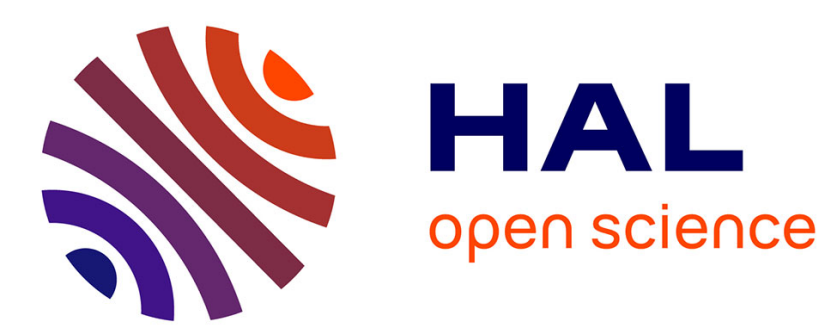

\title{
COMPORTEMENT DE PLAQUES ÉPAISSES À L'IMPACT NORMAL DE CYLINDRES
}

\author{
A. Neme, N. Dahan, S. Fouquet
}

\section{To cite this version:}

A. Neme, N. Dahan, S. Fouquet. COMPORTEMENT DE PLAQUES ÉPAISSES À L'IMPACT NORMAL DE CYLINDRES. Journal de Physique IV Proceedings, 1991, 01 (C3), pp.C3-247-C3-252. 10.1051/jp4:1991334 . jpa-00250476

\section{HAL Id: jpa-00250476 https://hal.science/jpa-00250476}

Submitted on 1 Jan 1991

HAL is a multi-disciplinary open access archive for the deposit and dissemination of scientific research documents, whether they are published or not. The documents may come from teaching and research institutions in France or abroad, or from public or private research centers.
L'archive ouverte pluridisciplinaire HAL, est destinée au dépôt et à la diffusion de documents scientifiques de niveau recherche, publiés ou non, émanant des établissements d'enseignement et de recherche français ou étrangers, des laboratoires publics ou privés. 
Colloque C3, suppl. au Journal de Physique III, Vol. 1, octobre 1991

\title{
COMPORTEMENT DE PLAQUES EPAISSES À L'IMPACT NORMAL DE CYLINDRES
}

\author{
A. NEME* ${ }^{*}$ N. DAHAN ${ }^{*}$ et $S$. FOUQUET $^{* *}$ \\ *Laboratoire de Mécanique et Technologie/CNRS/PARIS VI, ENS \\ de Cachan, 61 avenue du Président wilson, F-94230 Cachan, France \\ ${ }^{*}$ Etablissement Technique Central de I'Armement, CREA, 16 bis \\ avenue Prieur de la côte d'Or, F-94114 Arcueil, France
}

\begin{abstract}
The study of normal impact of cylinders on thick plates usually needs finite element calculations. It implies special softwares using and long-time solving. In order to reduce both calculation costs and the analysis field of this large problem, a new approach is performed. An analytical modelling is coupled with a numerical time integration of impact on isotropic rigid plastic metal plates by rigid rods. Impact velocity range is below $2000 \mathrm{~m} / \mathrm{s}$.

Modelling is based on the choice of a 2 -parameters continuum differentiable eulerien velocity field. It also fulfiled the material non compressibility, the kinematic boundary and stress boundary conditions according to the mechanical behaviour of the plate. A new extremum principle is developped and used to obtain the dimensions of the strained area in the plate. The energy balance of the plate-cylinder system leads to rod's acceleration.

Meanwhile, only penetration phenomenon is studied in this paper.
\end{abstract}

1 - Introduction.

L'analyse par éléments finis pour ce type de problème s'avère fort couteuse et l'utilisation des résuitats numéiques souvent difficile. Pour ces raisons des modélisations analytiques sont développées permettant une meilleure analyse des principaux phénomènes physiques rencontrés lors de l'impact avec une erreur raisonnable par rapport aux résultats expérimentaux.

Notre analyse s'est inspiré des travaux de Swanson-Donaldson [1], des travaux de Ravid-Bodner [2] et des travaux de Penazzi-Dahan [3].

Les travaux des premiers cités sont essentiellement de type monodimentionnel. Ils utilisent la conservation de la quantité de mouvement du projectile de façon à accéder à son accélération durant l'impact. Cette façon de faire permet une modélisation acceptable du comportement du projectile pour une gamme de vitesses d'impact de l'ordre de 1000 à $2000 \mathrm{~m} / \mathrm{s}$.

En ce qui concerne les travaux des seconds, ils sont beaucoup plus réalistes; d'une part leur modèle est bidimentionnel (axi-symétrie) et d'autre part ils utilisent le bilan en puissance de l'ensemble "plaqueprojectile" durant l'impact comme équation de conservation. Grâce à cela et suivant l'hypothèse d'un projectile indéformable, ils analysent de manière correcte la pénétration d'un cylindre pour des vitesses inférieures à $1000 \mathrm{~m} / \mathrm{s}$.

Les travaux des demiers cités diffèrent des deux précédents par le choix d'une description lagrangienne permettant cette fois ci un bilan énergétique mais ceci dans le cadre de l'impact de plaques minces.

Pour définir notre étude, nous avons conservé les idées de base des modèles de Ravid-Bodner et Penazzi-Dahan. En ce qui concerne l'optimisation d'une famille de champs de vitesse cinématiquement admissibles dans la plaque, nous utilisons une méthode variationnelle et un bilan énergétique afin de déterminer le mouvement du cylindre.

2 - Nouvelle approche: Méthode Inverse Analyse Globale (MIAG)

Le cylindre est supposé indéformable. La plaque (P) se comporte de façon isotrope rigide plastique écrouissable et est considérée infinie perpendiculairement à l'impact. 
Nous faisons choix d'une famille de champ de vitesse $\left(v^{\circ}\right)$ dépendant de deux paramètres $(\alpha, \eta)$ qui caractérisent la zone affectée (D) par l'impact.

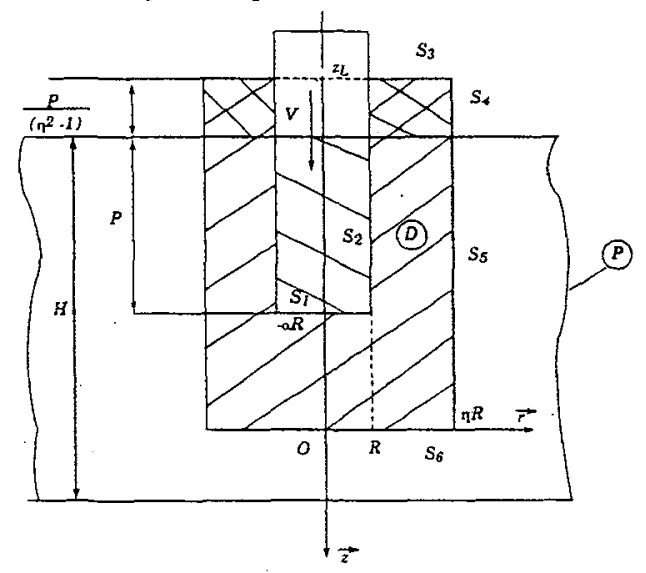

Figure 1: Configuration cylindre-plaque durant la pénétration.

Le choix de la zone affectée par l'impact (figure 1) permet de simplifier le calcul analytique du champ de vitesse $v^{*}$. En effet nous conférons à cette famille de champs les conditions suivantes.

$-v^{*}$ de classe $C^{1}$ sur $P$.

$-v^{*}=0$ sur $P \backslash D$.

$-\operatorname{div}\left(\mathbf{v}^{*}\right)=0$.

$-\mathrm{v}_{\mathrm{zz}}^{*}=\varphi_{1}(\mathrm{r}) \psi_{1}(\mathrm{z})+\varphi_{2}(\mathrm{r}) \psi_{2}(\mathrm{z})$.

$-\left\langle\mathbf{v}^{*} \mid \mathbf{z}\right\rangle=V$ et $\left\langle\mathbf{r} \mid \sigma^{\circ}(\mathbf{z})\right\rangle=0$ sur $S_{1}$.

$-\left\langle v^{*} \mid r\right\rangle=0$ et $\left\langle z \mid \sigma^{*}(r)\right\rangle=0$ sur $S_{2}$.

$-\left\langle\mathbf{r i \sigma}^{*}(\mathbf{z})\right\rangle=\left\langle\mathbf{z} \mid \sigma^{*}(\mathbf{z})\right\rangle=0$ sur $\mathrm{S}_{3}$.

$-<r\left|\sigma^{*}(\mathbf{r})\right\rangle=\langle z| \sigma^{*}(\mathbf{r})>=0$ sur $\mathrm{S}_{4}$.

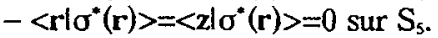

$-\left\langle\right.$ rl $\left.\sigma^{*}(\mathrm{z})\right\rangle=\left\langle z \mid \sigma^{*}(\mathrm{z})\right\rangle=0$ sur $\mathrm{S}_{6}$.

$<\mid>$ représente le produit scalaire dans l'espace vectoriel considéré.

Toutes ces conditions assurent à $\mathbf{v}^{*}$ d'être cinêmatiquement admissible et "statiquement" admissible aux frontières via la loi de comportement liant $\sigma^{*}$ à $v^{*}$ et au taux de déformations $\mathbf{D}^{*}$.

$\mathrm{Ci}$ dessous (figure $2,3,4$ et 5 ) nous donnons une représentation de $v_{r}^{*}(r, z) / V$ et de $^{*}{ }_{z}^{*}(r, z) / V$ dans la zone affectée (D), pour $(\alpha, \eta)=(1,2)$ et une profondeur de pénétration du cylindre égale à $R$.

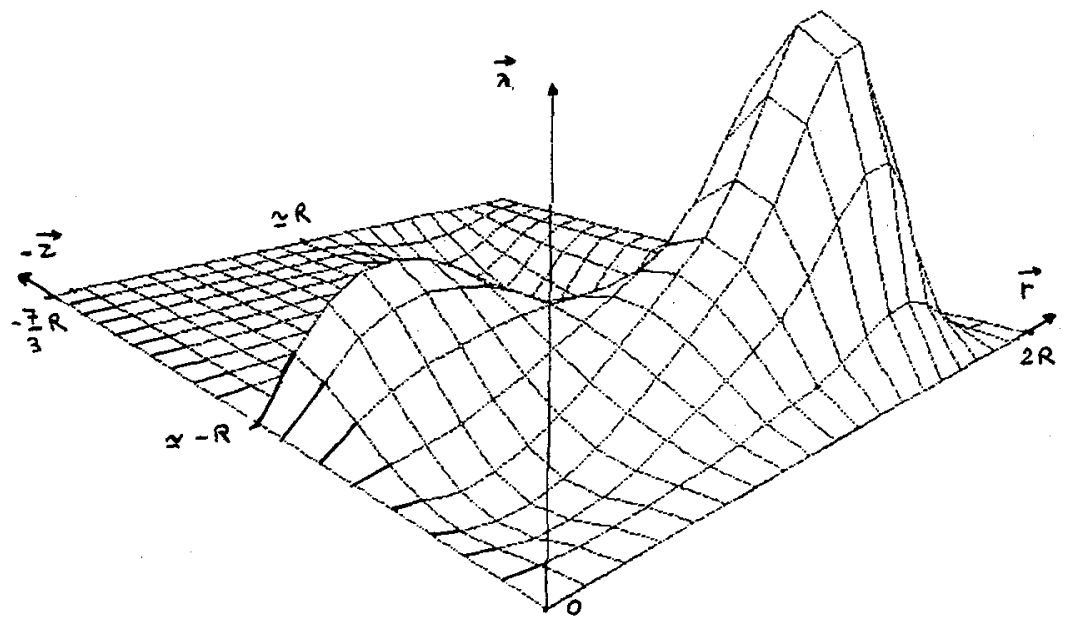

Figure 2: Représentation de $a=v_{r}^{*}(r, z) / V$ dans la zone (D) vue du point $z=R, r=-R, a=3 R$ 


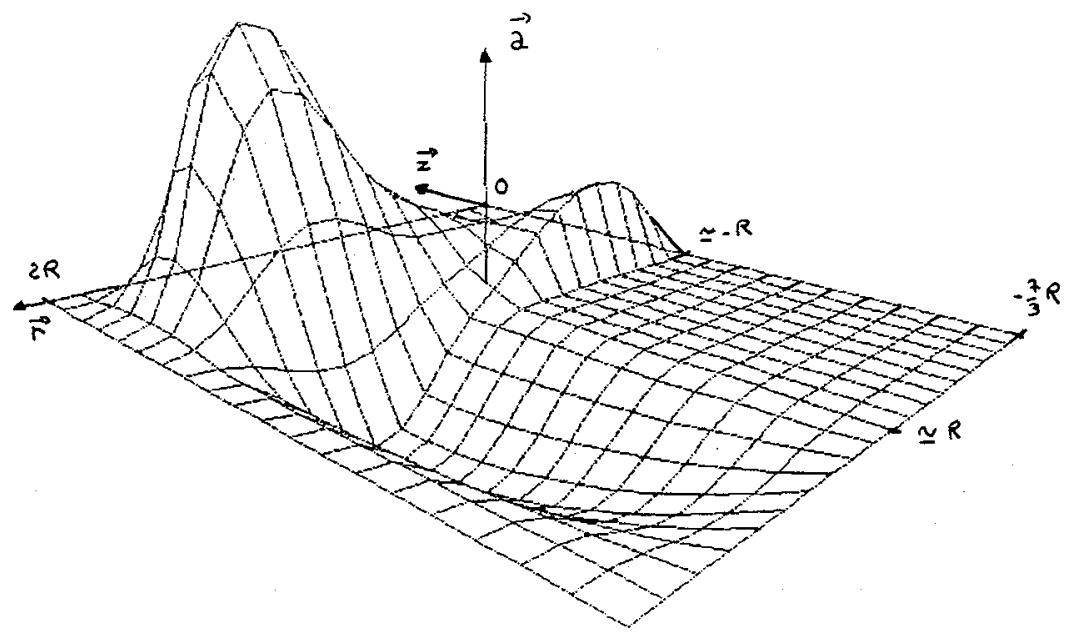

Figure 3: Représentation de $a=v_{r}^{*}(r, z) / V$ dans la zone (D) vue du point $z=-3 R, r=3 R, a=3 R$

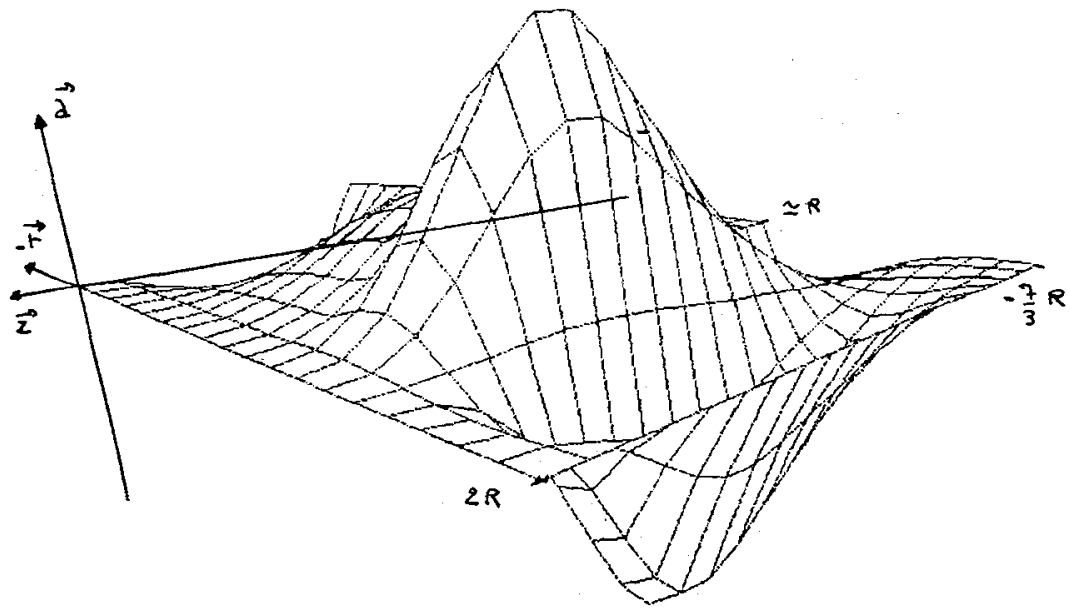

Figure 4: Représentation de $a=v_{z}{ }^{*}(r, z) / V$ dans la zone (D) vue du point $z=R, r=3 R, a=3 R$

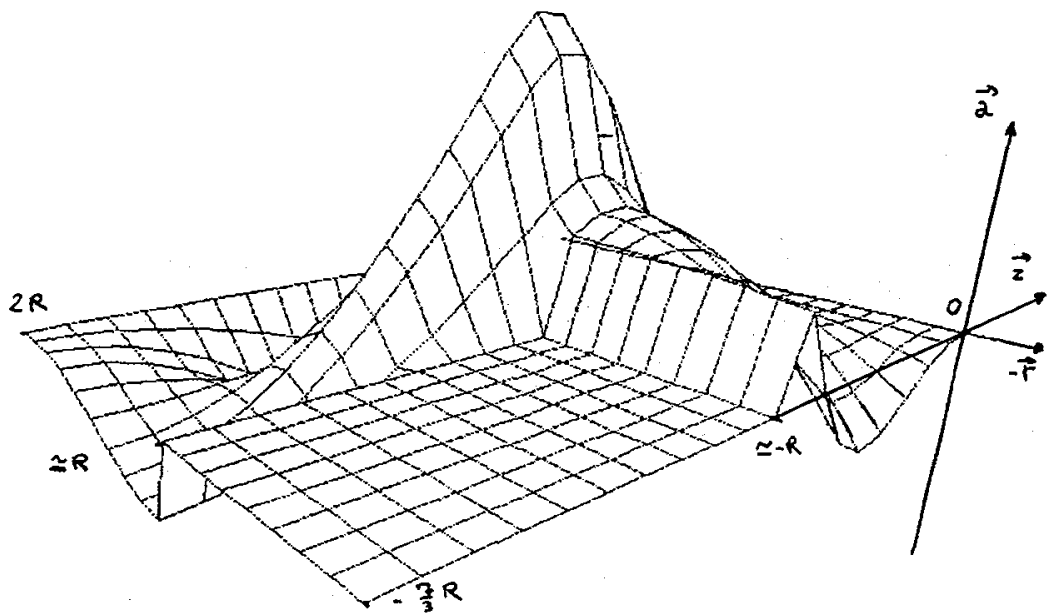

Eigure 5: Représentation de $a=v_{z}{ }^{*}(r, z) / V$ dans la zone (D) vue du point $z=-3 R, r=-R, a=3 R$ 
Dès lors il nous est possible de construire une fonctionnelle $F$ définie par la relation:

$$
F\left(v^{*}\right)=\int_{p}\left\|\operatorname{Div}^{T}\left(\sigma^{*}\right)+f-\varrho \Gamma\left(v^{*}\right)\right\| \operatorname{Vol}_{p}
$$

f sont les forces à distance (négligeables dans notre problème).

Q est la masse volumique de la plaque.

m point courant de la plaque.

$\Gamma\left(\mathbf{v}^{*}\right)$ est définie par:

$$
\Gamma\left(v^{*}\right)=\left(\partial v^{*} / \partial t\right)+\left(\partial v^{*} / \partial m\right)\left(v^{*}\right)
$$

Ainsi nous assurons que, quel que soit $v^{*}, F\left(v^{*}\right) \geq 0$ et que si $v^{*}=v$ (champ de vitesse réel) alors $F\left(v^{*}\right)=F(v)=0$. Nous avons donc une fonctionnelle qui permettra d'optimiser les paramètres $(\alpha, \eta)$ par rapport à la solution exacte. Remarquons que cette fonctionnelle sert aussi de quantificateur de "l'écart à l'équilibre" du champ $v^{*}$. Il est possible pour deux familles de champs de vitesse satisfaisant aux conditions aux limites de comparer leur erreur $(\mathrm{F})$ et de faire choix de la famille la plus proche du critère.

Enfin le bilan d'énergie-puissance donne accès à la valeur de l'accélération du cylindre:

$$
(d V / d t)=-\left[\int_{P}\left(\left\langle\sigma^{*} \mid D^{*}\right\rangle+Q\left\langle\Gamma\left(v^{*}\right) \mid v^{*}\right\rangle\right) V_{P o l}\right] /\left(m_{c} V\right)
$$

avec $m_{c}$ la masse du cylindre.

3 - Analyse expérimentale

Pour valider notre approche et la valeur des paramètres de la zone affectée, nous avons effectué des expériences d'impacts de plaques en AG5 (5083 h112) par des cylindres de diamètre $11 \mathrm{~mm}$, de longueur 16 $\mathrm{mm}$ en Acier 100C6 (Acier à roulement).

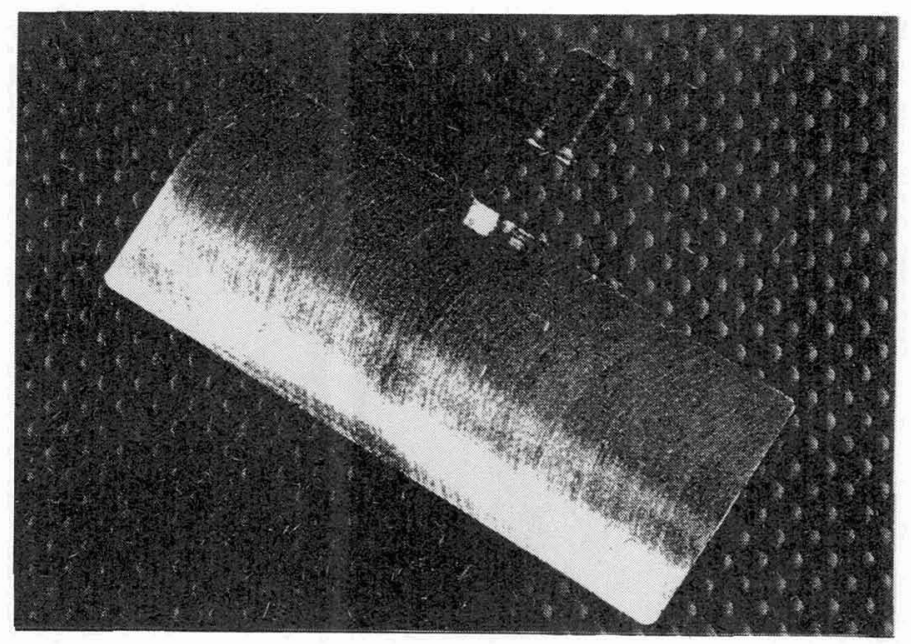

Figure 6: Vitesse d'impact $280 \mathrm{~m} / \mathrm{s}$, épaisseur de la plaque AG5 $40 \mathrm{~mm}$.

L'utilisation d'une caméra ultra-rapide nous a permis d'avoir une information sur la durée d'impact. Par exemple dans le cas de la figure 6 une durée de l'ordre de 25 à 30 microsecondes. Il sera possible d'accéder à l'évolution de la vitesse du cylindre jusqu'à l'arrêt du processus de déformation grâce à l'utilisation d'un système optique.

Une première analyse micrographique (figure 7) a permis, pour un impact sensiblement identique à 
celui présenté figure 6, d’avoir une estimation (figure 8) des dimensions $(\alpha, \eta)$ de la zone affectée (D).

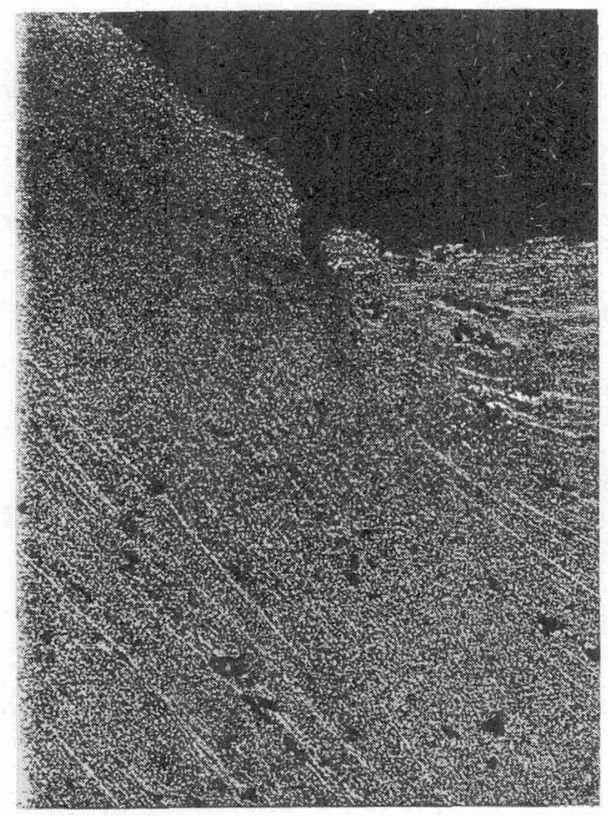

Figure 7: Coupe micrographique $(\times 400)$ autour du point $z=-\alpha R, r=-R$

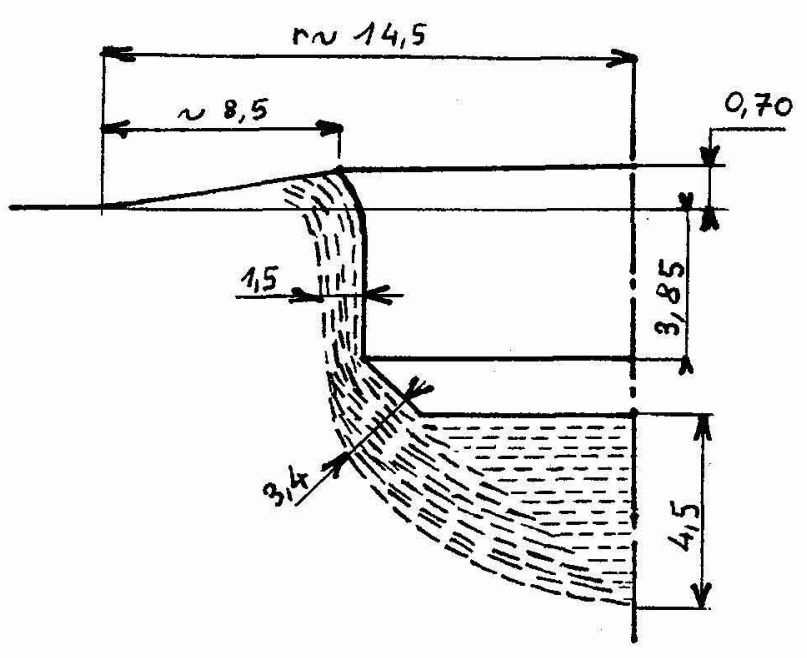

Figure 8: Estimation des dimensions de la zone affectée (D) en mm

$4-$ Conclusions

Des comparaisons sont en cours entre notre approche et les travaux de Ravid-Bodner. La première constatation actuellement met en évidence des résultats avec la méthode MIAG en bon accord avec les valeurs expérimentales.

Dans un avenir proche nous modéliserons le stade de la "perforation" en portant notre réflexion sur le choix d'un critère de rupture. 
5 - Bibliographie

[1] *CP DONALDSON, R.CONTILIANO, C.SWANSON

"The qualification of target materials using the integral theory of impact."

Aeronautical Research Associates of Princeton Inc., report $n^{\circ}$ ARAP-295, interim report $15 \mathrm{Mar} 76,15 \mathrm{De}$ 76, New Jersey 08540, December 1976.

*CP DONALDSON, C.SWANSON

"Application of the integral impact theory to modelling long-rod penetrators."

Aeronautical Research Associates of Princeton Inc., report $n^{\circ}$ ARAP-333, interim report 15 Dec 76, 15 No 77, New Jersey 08540, March 1978.

[2] M.RAVID, S.R.BODNER

"Dynamic perforation of viscoplastic plates by rigid projectiles."

Int J Engng Sci Vol 21, No 6, pp 577-591, 1983.

Faculty of Mechanical Engineering, Technion Israël Institute of Technology, Haïfa 32000, Israël.

[3] L.PENAZZI, N.DAHAN

"Modélisation de l'impact de plaques minces par des projectiles de petits calibres. Méthode inverse." Journal de Physique, C3, T49, pp 595-600. 